\title{
Symbolic and Non-Symbolic Number Processing in Children with Developmental
}

\section{Dyslexia}

\author{
Ulf Träff $_{\mathrm{a}}$, Annemie Desoete $\mathrm{b}, \&$ Maria Chiara Passolunghi c \\ ${ }^{a}$ Department of Behavioural Sciences and Learning, Linköping University, \\ Linköping, Sweden
}

${ }^{\mathrm{b}}$ Department of Experimental Clinical and Health Psychology, Ghent University, Henri Dunantlaan 2, B-9000 Ghent, Belgium (e-mail: annemie.desoete@Ugent.be)

${ }^{\mathrm{c}}$ Department of Life Sciences, University of Trieste, Via Weiss 21, Building W, University of Trieste, 34128 Trieste, Italy (e-mail: passolu@units.it).

Running headline: Dyslexia and number processing

Author note

Correspondence concerning this article should be addressed to Ulf Träff, Department of Behavioural Sciences and Learning, Linköping University.

E-mail: ulf.traff@liu.se

Postal address: Linköping University, Campus Valla, SE-581 83 Linköping, Sweden

Telephone number: +4613282103

Fax number: +4613149403

Acknowledgement

This research was supported by grant from the Swedish Research Council for Health, Working Life and Welfare (2010-0078) awarded to Ulf Träff 


\section{Abstract}

This study examined number processing in 10-year-olds with developmental dyslexia (DD). The phonological deficit and double deficit hypotheses imply that children with DD might have a connection deficit that affects their ability to establish links between number symbols and magnitude representations. The double deficit hypothesis also posits that symbolic number difficulties may emerge due to difficulties with processes underlying rapid automatic naming (RAN). The DD group displayed difficulties with symbolic number processing but not with non-symbolic number processing. However, the underlying processes of this access or connection deficit appeared not to be related to phonological awareness or RAN. The DD group displayed impaired arithmetic fluency and calculation that were accounted for by defective processes underlying RAN. In view of the triple-code model, children with DD have impaired verbal number codes or defective access to verbal number codes but an intact core magnitude representation.

Keywords: Dyslexia, number processing, access deficit, rapid automatic naming, phonological representations 


\section{Introduction}

Developmental dyslexia (DD) refers to a specific learning disorder characterized by a persistent deficit in accurate and/or fluent word recognition and/or by poor spelling (American Psychiatric Association, 2013). There are several hypotheses concerning the causes of DD. Defective phonological representations are considered to be one of the core problems of DD (e.g., Peterson \& Pennington, 2012; Vellutino, Fletcher, Snowling, \& Scanlon, 2004; Rasmus \& Ahissar, 2012). The indistinct phonological representations of individuals with DD hamper their ability to establish links between graphemes and phonemes. This grapheme-phoneme correspondence is a vital process to learn to read an alphabetic written language system (Rasmus et al., 2003; Snowling, 2000).

Another account of DD is the double deficit hypothesis (Wolf \& Bowers, 1999) stating that DD is due to two independent deficits: indistinct phonological representations and/or impairment in processes underlying rapid automatic naming (RAN; Torppa, Georgiou, Salmi, Eklund, \& Lyytinen, 2012). The double deficit hypothesis distinguishes three deficit subtypes, phonological deficit, and RAN deficit, and double deficit (combination of the two single core deficit subtypes) (Steacy, Kirby, Parrila, \& Compton, 2014; Wolf, Bowers, \& Biddle, 2000). People with the phonological deficit subtype have problems with phonological awareness, word decoding, and reading comprehension, but not with RAN. Those with the RAN deficit subtype have problems with RAN, verbal fluency, reading comprehension and reading under timed conditions but not with phonological awareness and word decoding. Those with the double deficit subtype have problem with all the aforementioned areas (Steacy et al., 2014; Torppa, Parrila, Niemi, Lerkkanen, Poikkeus, \& Nurmi, 2013).

Similar to reading, learning mathematics requires learning the language-based symbolic number system (e.g., number words; numerals) and connecting it to the innate non-symbolic number system (Butterworth, 2010; Dehaene 1992; Geary, 2004; von Aster \& Shalev, 2007). 
Children begin to acquire the language-based symbolic number system when learning to talk (Gelman \& Butterworth, 2005; Piazza, 2010; von Aster \& Shalev, 2007). It is assumed that children first learn the counting words by rote and connect them to the innate number system. Then they learn the Arabic numerals and connect them to the counting words and the innate number system (Carey, 2004; Dehaene, 2011; Geary, 2013; Le Corre \& Carey; 2007; von Aster \& Shalev, 2007). Empirical support of the assumption that children's learning of the symbolic number system depends on language skills has been provided by LeFevre et al. (2010; see also Krajewski \& Schneider, 2009). According to the Triple code model (Dehaene 1992; see also von Aster \& Shalev, 2007), children possess three interconnected number codes: 1) the innate analogue number representation used for number comparison, number estimation and approximate arithmetic, 2) a verbal number code used for counting, and establishing and retrieving arithmetic facts and 3) a visual Arabic number code used during written multi-digit calculation.

Theoretically inspired by the Triple-code model, an increasing number of researchers have examined mathematical skills in dyslexia (Simmons \& Singleton, 2008). This research provides evidence that individuals with dyslexia have difficulties with specific aspects of mathematics. Consistent with the Triple-code model, stating that arithmetic facts are represented via a phonological code, individuals with dyslexia display impaired arithmetic fact retrieval and/or fluency skills, presumably due to their indistinct phonological representations (De Smedt \& Boets, 2010; Göbel \& Snowling, 2010; Simmons \& Singleton, 2008; Träff \& Passolunghi, 2015; Vukovic, Lesaux, \& Siegel, 2010). In contrast, they show no evidence of weakness concerning approximate symbolic arithmetic assumed to rely on the innate analogue magnitude representation and visual Arabic number code (Göbel \& Snowling, 2010; Hanich, Jordan, Kaplan \& Dick, 2001). However, a few studies suggest that children with dyslexia also have problems with written multi-digit calculation, which is 
assumed to rely on the visual Arabic number code (Jordan, Hanich, \& Kaplan, 2003; Träff \& Passolunghi, 2015; Vukovic et al., 2010). This unexpected weakness is probably due to that efficient multi-digit calculation requires fast and accurate retrieval of number facts, which depend on a verbal-phonological code (Andersson, 2008; Ashcraft, 1992; 1995; McCloskey, Caramazza \& Basili, 1985; Träff, 2013; Träff \& Passolunghi, 2015).

The present study sought out to further expand our knowledge with respect to mathematical skills in dyslexia by examining if children with dyslexia displaying difficulties with number processing.

To date, few studies have examined number processing in individuals with DD. Göbel and Snowling (2010) examined symbolic number processing in adults with DD. They found that adults with DD performed symbolic number comparison as accurate and fast as the controls. The size of the numerical distance effect was also similar to the controls. In the De Smedt and Boets (2010) study, adults with dyslexia performed non-symbolic number comparison equal to the controls. These two studies suggest that adults with DD appear to have intact number processing skills. However, two recent studies indicate that children with DD have difficulties with symbolic (verbal, Arabic) number processing, but not non-symbolic number processing (Moll, Göbel, \& Snowling, 2015; Raddatz, Kuhn, Holling, Moll, \& Dobel, 2016). In Moll et al. (2015) children with DD displayed difficulties with verbal counting, dot-counting (5-7 dots range), identifying and transcoding orally presented one-digit and multi-digit numbers, and symbolic number comparison. The children in Raddatz et al. (2016) performed poorly in dotcounting (5-9 dots range), and transcoding orally presented numbers, but not in symbolic number comparison.

A feasible account of the contradictory findings concerning number processing in children with DD and adults with DD is that children have had less time and experience with the symbolic number system compared with adults. They might not have established efficient and 
automatized links between the number symbols and underlying magnitudes. In view of the phonological deficit hypothesis and the double deficit hypothesis, it is plausible that the defective grapheme-phoneme correspondence that characterizes children with dyslexia also affect their ability to connect the language-based symbolic number system, especially counting words, with the underlying analogue magnitude representation. Thus, both hypotheses predict that children with dyslexia should display difficulties with symbolic number comparison due to their indistinct phonological representations but not with non-symbolic number comparison because their magnitude representation is assumed to be intact. Moreover, they should display normal distance and problem size effects when performing symbolic number comparison as their magnitude representation is assumed to be unaffected. Indeed, an account of developmental dyscalculia, the access deficit hypothesis (Rousselle \& Noël, 2007), states that dyscalculia is caused by a defective connection between the symbols (e.g., counting words; digits) and the underlying magnitude representation (see also Wilson \& Dehaene, 2007).

The double deficit hypothesis also states that children with DD should have difficulties with processes underlying RAN, that is, the speed with which an individual names a series of highly familiar visual stimuli (Wolf et al., 2000). This seemingly simple task entails a number of processes such as attention; visual pattern identification; integration of visual information with stored orthographic and phonological representations; access and retrieval of phonological codes; and organization of articulatory output (see Norton \& Wolf, 2012 for a review). The question is whether a RAN deficit has any negative effects on the performance of basic mathematical tasks. In some studies, RAN has been found to predict arithmetic fluency (e.g., Koponen, Salmi, Eklund, \& Aro, 2013; Koponen et al., 2016) whereas other studies have failed to obtain such a connection (Heikkilä, Torppa, Aro, Närhi, \& Ahonen, 2015). Theoretically, a RAN deficit may hamper performance on all tasks involving speeded retrieval of information from visual numerical symbols (i.e., digits), even though no verbal 
response is required. If so, it predicts that children with DD should display difficulties with many of the mathematical tasks included in the study, especially symbolic number comparison.

As prior research shows that children with dyslexia have difficulties with specific aspects of mathematics, the present study included tasks tapping arithmetic fluency, calculation, and approximate arithmetic. The study also included tasks tapping phonological awareness, RAN, general processing speed, verbal working memory, and visual-spatial working memory. These tasks were selected because research shows that individuals with dyslexia are impaired on these functions (De Weerdt, Desoete, \& Roeyers, 2013a; 2013b; Fletcher et al., 1994; Helland \& Asbjørnsen, 2000; Menghini et al., 2010; Rasmus, 2004; Reiter, Reiter, Tucha, \& Lange, 2005; Stanovich, \& Siegel, 1994; Stein \& Walsh, 1997) or/and that they contribute to mathematical performance and development (e.g., Andersson, 2007; Berg, 2008; Bull, Espy, \& Wiebe, 2008; Geary, 2004; Passolunghi, Mammarella \& Altoè, 2008; Passolunghi \& Pazzaglia, 2004; Swanson, 1994; Träff, 2013).

\section{Material and methods}

\subsection{Participants}

In total, 20 fourth-graders with $\mathrm{DD}$ and 35 age-matched fourth-graders without learning disabilities participated in the study. They were recruited by means of a letter of consent that the children took home to the parents from school. All children were fluent speakers of Swedish, had normal or corrected-to-normal visual acuity, and no hearing loss. The selection of the 20 children with dyslexia was based on four criteria to comply with the definition of DD in DSM 5, that is, a severe, persistent, and specific learning disorder (American Psychiatric Association, 2013). First, the child should have received individually adapted special education instructions in reading and writing (i.e., Swedish) during the last year and at the time of the study but should 
never have received any special education instruction in any other subject. (cf. Andersson \& Östergren, 2012; Skagerlund \& Träff, 2016). Second, in grade three, the child should have passed the national assessment tests in mathematics administered by the Swedish National Agency for Education. The first and second criteria were important in order to exclude the possibility that some of the children with dyslexia also were low achievers in mathematics. Third, the child should not have had any neuropsychological disturbances (e.g., ADHD). Fourth, the child's score on a standardized word-decoding task (see below) had to be at or below the $10^{\text {th }}$ percentile of the test norms. The 35 children in the control group had to have worddecoding scores between the $15^{\text {th }}$ and the $85^{\text {th }}$ percentile and should never have received any special education instruction.

In addition to the word-decoding task, a text-reading task and a measure of fluid intelligence (Raven, 1976) were administered. Information regarding background variables and results on the reading tests and the Raven's test are presented in Table 1. The number of girls and boys in the two groups did not differ significantly. The children with dyslexia did not perform significantly different from the control children on Raven's matrices test but performed poorer on the text-reading task.

Table 1 here

\subsection{General procedure}

Testing was conducted in one group session and one individual session, each lasting approximately 2 hours. They were divided into three sessions of 40 minutes each with two breaks of 10 to 15 minute. The two sessions were performed within a months time. All testing was performed by two female experimenters, and the same test order was used for all children. Instructions were given orally, read aloud from a printed manuscript to ensure that every 
participant was given identical information. The following tasks were administered during the individual session: complex word repetition, color-naming, digit matching, visual-matrix span, word decoding, non-symbolic number comparison, phonological segment subtraction, symbolic number magnitude comparison, and approximate symbolic arithmetic. The remaining tasks were administered during the group session.

\subsection{Reading tasks}

2.3.1. Word-decoding (Elwér, Fridolfsson, Samuelsson, \& Wiklund, 2009). The child had to read from a sheet of paper as many words as possible from a list of 100 words, presented in four columns, during 45 seconds. The child performed an A-version and a B-version, beginning with the A-version. The instruction was to read as quickly as possible without making any errors. A stopwatch was used to keep track of time, and the experimenter registered each error. The number of correctly read words was used as the dependent measure.

2.3.2. Text reading (Malmquist, 1977). The child had to read a 600-word long story about a turtle and water buffalo stealing bananas from a gardener. Twenty sentences were missing a word. The task was to select the correct word from a multiple-choice of three words so the sentence was correct. Four minutes was the maximum performance time. The number of correctly completed sentences was used as dependent measure.

\subsection{Experimental tasks}

2.4.1. Phonological segment subtraction. This task taped phonological awareness (Taube, Torneus, \& Lundberg, 1984). The task was to determine which segment that had been removed from a word (i.e., What has been removed from the word "crocodile" if only croco remains?). 2.4.2. Color naming. Two sheets of paper containing $30 \mathrm{XXX}$ in red, green, blue, black, and yellow constituted the test material. The colored XXX were presented in two columns with 15 
in each column. The task was to name the $30 \mathrm{XXX}$ as quickly as possible without making any errors. A stopwatch was used to measure the total time it took to name the $30 \mathrm{XXX}$. The combined response times for the two sheets of paper was used as an index of RAN (Temple \& Sherwood, 2002).

2.4.3. Digit matching. This task taped general processing speed. The material consisted of a sheet of paper with 30 rows of digits, each row consisting of seven digits, with two identical digits. The task was to cross out the two identical digits on each row as fast and accurately as possible. The time needed to complete the task was used as an index of processing speed.

2.4.4. Complex word repetition. In this verbal working memory task (Östergren \& Träff, 2013), the child was orally presented with a sequence of words. The child had to decide whether each presented word was an animal or not by answering "yes" or "no" before the next word was presented. At the end of the sequence the child had to recall the words in correct serial order. The first span size used was two words, the next was three, and so forth. Half of the words in the sequences were animals. Testing stopped when the child failed both trials of the same span length. The longest sequence remembered correctly, plus 0.5 points if the child managed to recall both trials correctly on the same span size, was used as a measure of working memory span.

2.4.5. Visual-matrix span. This visual working memory task was administrated via the SuperLAB 4.5 software. A matrix made up of squares was presented; some of the squares contained two black dots. The first task was to decide whether these dots were of equal size, and press the "*” key if they were equal or the " $A$ " key if they were not. The child had 3 seconds to respond, after which two additional dots appeared in another square while the former two dots were still visible. The second task was to remember the location of the dots in the matrix. When a sequence of dots had been presented, the matrix was removed, and the child was required to draw a cross in the correct squares on an identical empty matrix 
presented on a sheet of paper. The first matrix had $3 \times 3$ squares and two squares with black dots (i.e., span size two). The next matrix had $3 \times 4$ squares, and three squares with black dots. In this way, the complexity of the matrixes increased for each new span size. Testing stopped when the child failed both trials. The same scoring procedure as in the complex word repetition task was used.

2.4.6. Symbolic number comparison. This task taped the ability to quickly access the underlying magnitude representations of Arabic numerals (i.e., digits; Rousselle \& Noël, 2007). Two digits were simultaneously displayed on the computer screen. The task was to decide, as quickly as possible without making any errors, which of the two digits was the numerically larger one. Prior to each problem a "cross" was displayed in the center of the screen for $1000 \mathrm{~ms}$. The child responded by pressing the key corresponding to the appropriate side of the screen. The digits were displayed until the child responded. The test material consisted of one-digit and two-digit numbers that were presented in two separate blocks, starting with the one-digit block. Two distances were used, 1 (1-2, 5-6, 8-9, 21-22, 34-33, 74-73, 92-91) and 4-5 (1-6, 3-8, 4-9, 3-7, 31-36, 54-59, 68-63, 97-92) and each digit pair was presented twice (e.g., 2-3 and 3-2), resulting in a total of 32 trials for each block. Mean response times for correct responses were used as dependent measures. Error rates were low (5\%).

2.4.7. Non-symbolic number comparison. This task taped speed of access to, and acuity of the core number representation system. The Panamath software version 1.21 , developed by Halberda, Mazzocco and Feigenson (2008) administrated this task. Two arrays of randomly arranged dots (blue/yellow) ranging from 5 to 21 were simultaneously displayed on the computer screen. The task was to decide, as quickly as possible without making any errors, which of the two arrays contained more dots. The child responded by pressing the F or L key. The children had an unlimited amount of time to indicate their responses, but the stimuli was only presented for only $1382 \mathrm{~ms}$. Prior to each trial a fixation cross was displayed in the center 
of the screen. The child had to press the space bar to enable the next trial. One hundred and twelve trials distributed over four ratios $(1.21 ; 1.35 ; 1.56 ; 2.56)$, were presented. Two practice trials preceded the experimental trials. Fifty percent of the trials contained more blue dots than yellow dots. To ensure that attention was focused on numerosity, for $50 \%$ of the trials, the total blue and yellow surface areas were equal, and the dots varied in size. For each child, the program calculated mean response time and a Weber fraction value $(w)$ based on accuracy at each ratio. The $w$ value is an estimate of the acuity of the core number magnitude representation system (Halberda \& Feigenson, 2008).

2.4.8. Arithmetic fluency. In this paper-and-pencil test, the task was to solve as many singledigit addition and subtraction problems as possible during 2 minutes. The addition and subtraction problems were presented on two separate sheets of paper containing three columns with 27 problems in each column. The child responded in writing and was allowed 1 minute for each sheet of paper. All children began with addition.

2.4.9. Multi-digit calculation task. The child was instructed to solve twelve arithmetic problems (e.g., $568+421,824-488$ ) in 8 minutes. The problems were horizontally presented and became increasingly more difficult. The children responded in writing. All problems, except two, involved regrouping.

2.4.10. Approximate arithmetic. The material consisted of 24 two-digit arithmetic problems presented in one addition block and one subtraction block, starting with the addition block. For each trial, an arithmetic problem $(31+27)$ with two proposed answers (e.g., 60 and 48) was presented underneath the problem, one on the left and one on the right side. The task was to choose the answer closest to the correct answer without calculating. The child responded by pressing the key corresponding to the appropriate side of the screen. If the child did not respond within 5 seconds, the answer was considered incorrect, and the child was prompted to respond quicker next time (Hanich et al., 2001). The number of correctly solved combinations with 
response times within 5 seconds and mean response time were used as the dependent measures. However, the mean accuracy of the two groups was at the level of chance (DD: $M=12.90$; Controls; $M=13.83$ ), indicating that task was too difficult for children at this age to be sensitive enough to detect group differences. The task was therefore not further analyzed.

\section{Results}

Means, standard deviations, and reliability indexes for all measures are displayed in Table 2. Correlations among the tasks are displayed in Table 3. Analysis of variance (ANOVA) and regression analysis were used to examine group differences.

\subsection{Cognitive tasks}

As expected, the DD children performed the phonological segment subtraction task and color-naming task significantly worse than the controls (see Table 2). They also performed slower on the digit-matching task (see Table 2). No significant group differences emerged on the working memory tasks.

Table 2 and 3 here

\subsection{Number processing}

Two 2 (groups) $\times 2$ (numerical distance) mixed ANOVAs were computed on the RT measures of symbolic number comparison tasks.

On the one-digit number task, a significant group effect, $F(1,53)=8.86, p=.004, \omega^{2}=.13$, emerged, and a significant distance effect, $F(1,53)=89.18, p<.001, \omega^{2}=.62$, emerged but no interaction effect $(p=.104)$ was observed. 
On the two-digit number task, a significant group effect, $F(1,53)=6.31, p=.015, \omega^{2}=.09$, emerged but not distance $(p=.097)$, or interaction effects $(p=.104)$. Thus, the DD group performed significantly slower than the controls on both tasks but displayed the distance effects to the same extent as the controls.

A mixed ANOVA was performed on the overall performance of the one- and two-digit number tasks to examine if the DD group displays a larger or smaller problem-size effect compared with the controls (i.e., group $\times$ problem-size effect). A significant problem-size effect, $F(1,53)=223.26, p<.001, \omega^{2}=.80$, emerged but not a group $\times$ problem-size effect interaction $(p=.128)$.

ANOVAs performed on the $w$-measure and the response time measure of the nonsymbolic number comparison task revealed that the DD group performed similar to the controls on both measures.

Can the slower performance of the DD group on the symbolic number comparison tasks be accounted for by their poor performance on the phonological segment subtraction and colornaming tasks? Two hierarchical multiple regression analyses were computed to address this question. The two cognitive tasks were entered in the first block and the group variable $($ dyslexia $=0$ vs. controls $=1)$ in the second block.

On the one-digit number task, the first block accounted for $14 \%, R^{2}=.14, F(2,52)=4.14$, $p=.022$, of the variation. The color-naming task, $\beta=.36, p=.008$, emerged as significant predictor, but not the phonological segment subtraction task $(p=.635)$. The group variable accounted for additional variance, $\Delta R^{2}=.09, F_{\text {change }}(1,51)=6.09, p=.017$. The phonological segment subtraction task and the color-naming task were not significant predictors ( $p s>.05$ ), when the full model was considered.

On the two-digit number task, the first block accounted for a significant amount of variance, $R^{2}=.13, F(3,51)=3.79, p=.029$. The color-naming task, $\beta=.35, p=.010$, 
emerged as a significant predictor, but not the phonological segment subtraction task $(p=$ $.805)$. The group variable accounted for additional variance, $\Delta R^{2}=.13, F_{\text {change }}(1,51)=8.60$, $p=.005$. None of the two cognitive tasks accounted for any unique variance $(p s>.05)$. Thus, the DD group still performed symbolic number comparisons significantly slower than the controls.

\subsection{Arithmetic tasks}

Compared with the controls, the DD children solved significantly fewer problems when performing the arithmetic fluency, $F(1,53)=8.70, p=.005, \omega^{2}=.12$, and the calculation tasks, $F(1,53)=15.64, p<.001, \omega^{2}=.21$.

To examine if the lower performance of the DD children on the arithmetic fluency and calculation tasks can be accounted for by their poor performance on the phonological segment subtraction task, and the color-naming task hierarchical multiple regression analyses were computed.

On the arithmetic fluency task, the first block accounted for a significant amount of variance, $R^{2}=.21, F(2,52)=6.74, p=.002$. The color-naming task, $\beta=-.41, p=.002$, emerged as a significant predictor, but not the phonological segment subtraction task $(p=$ .229). The group variable did not account for any additional variance $(p=.135)$. The colornaming task remained a significant predictor even when the full model was considered, $\beta=-$ $.34, p=.015$.

On the calculation task, the first block accounted for a significant amount of variance, $R^{2}=$ $.30, F(2,52)=11.24, p<.001$. Both the phonological segment subtraction task, $\beta=.25, p=$ .040 , and the color-naming task, $\beta=-.46, p<.001$, turned out as significant predictors. The group variable did not account for any additional variance $(p=.064)$. The color-naming task remained a significant predictor even when the full model was considered, $\beta=-.38, p=.004$. 
An additional hierarchical multiple regression analysis was computed to examine if the color-naming task will remain a significant predictor even when the arithmetic fluency task is entered into the model. The first block accounted for $40 \%, R^{2}=.40, F(3,51)=11.60, p<$ .001 , of the variation in calculation. The arithmetic fluency task, $\beta=.36, p=.004$, and the color-naming task, $\beta=-.31, p=.012$, were significant predictors, but not the phonological segment subtractions task, $\beta=.19, p=.088$. The group variable did not account for any additional variance, $\Delta R^{2}=.02, F_{\text {change }}(1,50)=1.98, p=.166$. The arithmetic fluency task, $\beta$ $=.33, p=.011$, and the color-naming task, $\beta=-.27, p=.036$, were significant predictors, but not the phonological segment subtraction task, $\beta=.06, p=.707$.

\section{Discussion}

This study examined if children with DD display difficulties with number processing. Two accounts of why they might have this problem were tested. In view of the phonological deficit hypothesis, it was assumed that children with DD suffer from impaired accessibility or mapping between the number symbols and their underlying magnitude representations due to indistinct phonological representations. Thus, they should have problems with symbolic number comparison but not non-symbolic number comparison. The double deficit hypothesis posits that children with DD should display difficulties with symbolic number comparison due to indistinct phonological representations and/or difficulties with processes underlying RAN.

The present findings were consistent with the phonological deficit hypothesis and double deficit hypothesis as the DD children performed the symbolic number comparison tasks slower than the controls. Thus, this study provides further evidence that children with DD (and no dyscalculia) might also have impaired symbolic number processing. In contrast to studies on adults with dyslexia (Göbel \& Snowling, 2010) but in line with Moll et al. (2015; 
see also De Weerdt et al., 2013b), children with dyslexia appear to suffer from an access deficit, a defective connection between the number symbols (e.g., digits) and their corresponding magnitude representations (Noël \& Rousselle, 2011; Wilson \& Dehaene, 2007). This difference between adults and children with dyslexia may be explained by the fact that the children with DD have had less experience with the symbolic number system and thereby less time to establish adequate number symbol-number magnitude correspondence compared with adults with DD. The present study also corroborates and extends findings reported by Göbel and Snowling (2010) and De Smedt and Boets (2010) as the children with dyslexia displayed normal distance and problems size effects when performing symbolic number comparison and performed non-symbolic number comparison as fast and accurately as the controls. These findings suggest that the children with DD probably have an intact core number magnitude system (De Smedt \& Boets, 2010).

Even though the two hypotheses were supported by the results of symbolic number comparison task, the results of the hierarchical regression analyses provide further important and novel findings. The slower performance of the DD children on the symbolic number comparison tasks remained even after accounting for their poorer performance on the phonological segment subtraction and the color-naming tasks. Indeed, including these two tasks in the regression model had no effect on the group variable suggesting that the DD children's connection problems are not related to processes tapped by the phonological segment subtraction or the color-naming tasks. Future studies should be aimed at pinpointing the defective mechanism underlying the slower symbolic number comparison of children with DD.

The Triple-code model asserts that arithmetic facts are represented via a verbal code (Dehaene, 1992). Consistent with this and prior studies, the children with DD solved fewer problems when performing the arithmetic fluency task (De Smedt \& Boets, 2010; Göbel \& 
Snowling, 2010; Vukovic et al., 2010). However, accounting for the color-naming and phonological segment subtraction tasks via multiple regression analysis eliminated the poorer arithmetic fluency performance of the DD group. The color-naming task accounted for unique variance but not the phonological segment subtraction task.

The Triple-code model, states that a visual Arabic number code is used during written multidigit calculation. Therefore, it was unexpected to find that the DD group displayed an inferior performance on the multi-digit calculation task compared with the controls. However, impaired calculation in dyslexia has previously been reported by Vukovic et al. (2010) and Träff and Passolunghi (2015). The results from the multiple regression analyses revealed that the poor calculation performance of the DD children was fully accounted for by their poor performance on the color-naming and phonological segment subtraction tasks (Andersson, 2008; Ashcraft, 1995; McCloskey et al., 1985; Träff, 2013). The color-naming task accounted for unique variance in calculation even when the arithmetic fluency task was entered into the model, but the phonological segment subtraction task did not (cf. Koponen et al., 2013; 2016).

The new findings in relation to the arithmetic fluency and the calculation tasks are consistent with the double deficit hypothesis, suggesting that defective processes underlying RAN impair arithmetic fluency and calculation in children with DD.

\section{Conclusions, future research, and limitations}

This study provides for the first time evidence that children with DD (and no dyscalculia) have impaired symbolic number processing skills due to a reduced accessibility or connection between numerical symbols and the underlying magnitude representation. However, the underlying processes of this access deficit appear not to be related to phonological awareness or RAN. It is a task for future studies to pinpoint the defective mechanism underlying the slower symbolic number comparison of children with DD. 
Similar to prior research, children with DD displayed intact non-symbolic number processing, suggesting that they have an intact core number magnitude representation system. Consistent with previous studies, the children with DD also demonstrated impaired arithmetic fluency and calculation that seem to be connected to defective processes underlying RAN.

The present results should be interpreted with care, because there are some limitations to the study. First, as already mentioned only 20 fourth-grade children with DD in Sweden were tested. As Sweden has a very transparent reading and spelling system, the question is to what extent the present findings can be generalized to countries with less transparent orthography. Furthermore, the combination of using a time constraint, word-decoding task to classify children DD, which is appropriate in transparent orthography, and using time constraint number processing and mathematical tasks may reduce the generalizability of the findings even more. Thus, additional research is most needed in other countries (with a less transparent orthography) and in other age groups. Second, studies on children with combined dyslexia and dyscalculia should be included to obtain a complete overview of this issue. These two limitations indicate that only a part of the picture was investigated, so additional studies should focus on these aspects.

Third, two tasks used were not completely calibrated for the study. The digit-matching task was used to tap general processing speed. It would have been more appropriate to use another type of stimuli instead of digits (e.g., abstract figures) as the key outcome variables were mathematics. There is a risk that the Panamath task used to tap non-symbolic number comparison was too easy, as indicated by the low mean $w$-values for the two groups (DD: .23; Controls: .25) compared with test norm mean $w$-value of .31 available on the Panamath web page (Halberda et al., 2008). This task may be too easy in order to be sensitive enough to detect group differences. However, the Panamath task with the same settings as used in the present study has been sensitive enough to detect impaired non-symbolic number comparison 
in third-graders with developmental dyscalculia (Olsson, Östergren, \& Träff, 2016).

Furthermore, although the mean $w$-values were low, neither group displayed signs of ceiling effect, the $w$-values ranged from $w=.12$ to $w=.77$, which would be expected if the settings were too easy. Future studies should despite this make an effort to use non-symbolic number comparison tasks with age-appropriate settings to avoid ceiling and floor effects.

Nevertheless, the present findings indicate that it is important to examine symbolic number processing in children with DD as they might have difficulties with this task, as well as arithmetic fact retrieval and calculation. An important task for future research would be to examine if phonological intervention programs aiming at improving reading skills of children with DD also have positive effects on these children's symbolic number processing and arithmetic skills. 


\section{References}

American Psychiatric Association. (2013). Diagnostic and statistical manual of mental disorders ( $5^{\text {th }}$ ed.). Arlington, VA: American Psychiatric Publishing.

Andersson, U. (2007). The contribution of working memory to children's mathematical word problem solving. Applied Cognitive Psychology, 21, 1201-1216.

Andersson, U. (2008). Mathematical competencies in children with different types of learning difficulties. Journal of Educational Psychology, 100, 48-66.

Andersson, U., \& Östergren, R. (2012). Number magnitude processing and basic cognitive functions in children with mathematical learning disabilities. Learning and Individual Differences, 22, 701-714.

Ashcraft, M. H. (1992). Cognitive arithmetic: A review of data and theory. Cognition, 44, 75106.

Ashcraft, M. H. (1995). Cognitive psychology and simple arithmetic: A review and summary of new directions. Mathematical Cognition, 1, 3-34.

Berg, D. H. (2008). Working memory and arithmetic calculation in children: The contributory roles of processing speed, short-term memory, and reading. Journal of Experimental Child Psychology, 99, 288-308.

Bull, R., Espy, K. A., \& Wiebe, S. A. (2008). Short-term memory, working memory, and executive functioning in preschoolers: Longitudinal predictors of mathematical achievement at age 7 years. Developmental Neuropsychology, 33, 205-228.

Butterworth, B. (2010). Foundational numerical capacities and the origins of dyscalculia. Trends in Cognitive Sciences, 14, 534-541.

Carey, S. (2004). Bootstrapping and the origin of concepts. Daedalus, 59-68.

Dehaene, S. (1992). Varieties of numerical abilities. Cognition, 44, 1-42.

Dehaene, S. (2011). The number sense. New York: Oxford University Press. 
De Smedt, B., \& Boets, B. (2010). Phonological processing and arithmetic fact retrieval: Evidence from developmental dyslexia. Neuropsychologia, 48, 3973-3981.

De Weerdt, F., Desoete, A., \& Roeyers, H. (2013a). Working Memory in Children With Reading Disabilities and/or Mathematical Disabilities. Journal of Learning Disabilities, 46, 461-472. DOI: 10.1177/0022219412455238

De Weerdt, F., Desoete, A., \& Roeyers, H. (2013b). Behavioral inhibition in children with learning disabilities. Research in Developmental Disabilities, 34, 1998-2007. doi:10.1016/j.ridd.2013.02.020

Elwér, Å., Fridolfsson, I., Samuelsson, S. \& Wiklund, C. (2009). LäSt - Test of reading and spelling for grade 1-5. Linköping: Vasa Tryck AB.

Fletcher, J. M., Shaywitz, S. E., Shankweiler, D. P., Katz, L., Liberman, I. Y., \& Stuebing, K. K. (1994). Cognitive profiles of reading disability: Comparisons of discrepancy and low achievement definitions. Journal of Educational Psychology, 86, 6-23.

Geary, D. C. (2004). Mathematics and learning disabilities. Journal of Learning Disabilities, $37,4-15$.

Geary, D. C. (2013). Early Foundations for Mathematics Learning and Their Relations to Learning Disabilities. Current Directions in Psychological Science, 22, 23-27.

Gelman, R., \& \& Butterworth, B (2005). Number and language: How are they related? Trends in Cognitive Sciences, 9, 6-10.

Göbel, S., M., \& Snowling, M., J. (2010): Number-processing skills in adults with dyslexia, The Quarterly Journal of Experimental Psychology, 63, 1361-1373.

Halberda, J. and Feigenson, L. (2008). Developmental change in the acuity of the 'Number Sense': The Approximate Number System in 3-, 4-, 5-, and 6-year-olds and adults. Developmental Psychology. 44, 1457-1465. 
Halberda, J., Mazzocco, M.M.M, \& Feigenson, L. (2008). Individual differences in non-verbal number acuity correlate with maths achievement. Nature 455, 665-668.

Hanich, L. B., Jordan, N. C., Kaplan, D., \& Dick, J. (2001). Performance across different areas of mathematical cognition in children with learning disabilities. Journal of Educational Psychology, 93, 615-626.

Heikkilä, R., Torppa, M., Aro, M., Närhi, V., \& Ahonen, T. (2015). Double-deficit hypothesis in a clinical sample: extension beyond reading. Journal of Learning Disabilities. First published on February 25, 2015. doi:10.1177/0022219415572895

Helland, T., \& Asbjørnsen, A. (2000). Executive functions in dyslexia. Child Neuropsychology, $6,37-48$.

Jordan, N. C., Hanich, L. B., \& Kaplan, D. (2003). A longitudinal study of mathematical competencies in children with specific mathematics difficulties versus children with comorbid mathematics and reading difficulties. Child Development, 74, 834-850.

Koponen, T., Salmi, P., Eklund, K., \& Aro, T. (2013). Counting and RAN: Predictors of arithmetic calculation and reading fluency. Journal of Educational Psychology, 105(1), 162175. doi:10.1037/a0029285

Koponen, T., Salmi, P., Torppa, M., Eklund, K, Aro, T., Aro, T., Poikkeus, A-M., Lerkkanen, M-K., \& Nurmi, J-E. (2016). Counting and rapid naming predict the fluency of arithmetic and reading skills. Contemporary Educational Psychology, 44-45, 83-94. doi:10.1016/j.cedpsych.2016.02.004

Krajewski, K., \& Schneider, W. (2009). Exploring the impact of phonological awareness, visual-spatial working memory, and preschool quantity-number competencies on mathematics achievement in elementary school: findings from a 3-year longitudinal study. Journal of Experimental Child Psychology, 103, 516-531. doi: 10.1016/j.jecp.2009.03.009 
Le Corre, M., \& Carey, S. (2007) One, two, three, four, nothing more: An investigation of the conceptual sources of the verbal counting principles. Cognition, 105, 395-438. doi: 10.1016/j.cognition.2006.10.005

LeFevre, J.-A., Fast, L., Skwarchuk, S.-L., Smith-Chant, B. L., Bisanz, J., Kamawar, D., \& Penner-Wilger, M. (2010). Pathways to mathematics: longitudinal predictors of performance. Child Development, 81(6), 1753-67. doi:10.1111/j.1467-8624.2010.01508.x

Malmquist, E. (1977). Reading and writing difficulties in children. Analysis and treatment. Lund: Gleerups.

McCloskey, M., Caramazza, A., \& Basili, A. (1985). Cognitive mechanisms in number processing and calculation: Evidence from dyscalculia. Brain and Cognition, 4, 171-196.

Menghinia, D., Finzi, A., Benassic, M., Bolzanic, R., Facoetti, A., Giovagnolic, S., Ruffino, M., Vicari, S. (2010). Different underlying neurocognitive deficits in developmental dyslexia: A comparative study. Neuropsychologia, 48, 863-872.

Moll, K., Göbel, S. M., \& Snowling, M. J. (2015). Basic number processing in children with specific learning disorders: Comorbidity of reading and mathematics disorders. Child Neuropsychology, 21, 399-417. DOI: 10.1080/09297049.2014.899570

Noël, M.-P., \& Rousselle, L. (2011). Developmental Changes in the Profiles of Dyscalculia: An Explanation Based on a Double Exact-and-Approximate Number Representation Model. Frontiers in human neuroscience, 5, 165.

Norton, E. S., \& Wolf, M. (2012). Rapid automatized naming (RAN) and reading fluency: Implications for understanding and treatment of reading disabilities. Annual Review of Psychology, 63, 427-452. Doi: 10.1146/annurev-psych-120710-100431

Olsson, L., Östergren, R., \& Träff, U. (2016). Developmental Dyscalculia: A deficit in the approximate number system or an access deficit? Cognitive Development, 39, 154-167 DOI: 10.1016/j.cogdev.2016.04.006 
Östergren, R., \& Träff, U. (2013). Early number knowledge and cognitive ability affect early arithmetic ability. Journal of Experimental Child Psychology, 115, 405-421.

Passolunghi, M. C., Mammarella I. C., \& Altoè, G. (2008). Cognitive abilities as precursors of the early acquisition of mathematical skills during first through second grades. Developmental Neuropsychology, 33, 229-250.

Passolunghi, M. C., \& Pazzaglia, F. (2004). Individual differences in memory updating in relation to arithmetic problem solving. Learning and Individual Differences, 14, 219-230.

Peterson, R. L., \& Pennington, B. F. (2012) Developmental dyslexia. Lancet, 379, 1997-2007. doi: 10.1016/S0140-6736(12)60198-6.

Piazza, M. (2010). Neurocognitive start-up tools for symbolic number representations. Trends in Cognitive Sciences, 14, 542-551.

Raddatz, J., Kuhn, J.-T., Holling, H., Moll, K., \& Dobel, C. (2016). Comorbidity of arithmetic and reading disorder: Basic number processing and calculation in children with learning impairments. Journal of Learning Disabilities, 1-11. DOI: 10.1177/0022219415620899

Ramus, F., \& Ahissar, M. (2012) Developmental dyslexia: The difficulties of interpreting poor performance, and the importance of normal performance. Cognitive

Neuropsychology, 29, 104-122. DOI: 10.1080/02643294.2012.677420

Ramus, F., Rosen, S., Dakin, S. C., Day, B. L., Castellote, J. M., White, S., et al. (2003). Theories of developmental dyslexia: Insights from a multiple case study of dyslexic adults. Brain, 126, 841-865.

Raven, J. C. (1976). Standard progressive matrices. Oxford: Oxford Psychologists Press.

Reiter, T., Reiter, A., Tucha, O., \& Lange, K. W. (2005). Executive functions in children with dyslexia. Dyslexia, 10, 1-16. 
Rousselle, L., \& Noël, M-P. (2007). Basic numerical skills in children with mathematics learning disabilities: A comparison of symbolic vs non-symbolic number magnitude processing. Cognition, 102, 361-395.

Simmons, F. R., \& Singleton, C. (2008). Do weak phonological representations impact on arithmetic development? A review of research into arithmetic and dyslexia. Dyslexia, 14, 77-94.

Skagerlund, K., \& Träff, U. (2016). Number processing and heterogeneity of developmental dyscalculia: Subtypes with different cognitive profiles and deficits. Journal of Learning Disabilities, 49, 36-50. DOI: 10.1177/0022219414522707

Snowling, M. J. (2000). Dyslexia (2nd ed.). Malden, MA: Blackwell Publishers.

Stanovich, K. E., \& Siegel, L. S. (1994). Phenotypic performance profile of children with reading disabilities: A regression-based test of the phonological-core variable difference model. Journal of Educational Psychology, 86, 24-53.

Steacy, L. M., Kirby, J. R., Parrila, R., \& Compton, D. (2014). Classification of double deficit groups across time: An analysis of group stability from kindergarten to second grade. Scientific Studies of Reading, 18, 255-273. DOI: 10.1080/10888438.2013.873936

Stein, J., \& Walsh, V. (1997). To see but not to read, the magnocellular theory of dyslexia. Trends in Neuroscience, 20, 147-152.

Swanson, H. L. (1994). Short-term memory and working memory: Do both contribute to our understanding of academic achievement in children and adults with learning disabilities? Journal of Learning Disabilities, 27, 34-50.

Taube, K., Tornéus, M., \& Lundberg, I. (1984). UMESOL: Phonological awareness. Guide to the identification and development. Stockholm: Psychology Publishing House. 
Temple, C. M., \& Sherwood, S. (2002). Representation and retrieval of arithmetical facts: Developmental difficulties. Quarterly Journal of Experimental Psychology A: Human Experimental Psychology, 55A, 733-752.

Torppa, M., Georgiou, G., Salmi, P., Eklund, K., \& Lyytinen, H. (2012). Examining the doubledeficit hypothesis in an orthographically consistent language. Scientific Studies of Reading, 16, 287-315. DOI: 10.1080/10888438.2013.873936

Torppa, M., Parrila, R., Niemi, P., Lerkkanen, M-K., Poikkeus, A-M., \& Nurmi, J-E. (2013). The double deficit hypothesis in the transparent Finnish orthography: a longitudinal study from kindergarten to Grade 2. Read and Writing, 26, 1353-1380. DOI 10.1007/s11145-0129423-2

Träff, U. (2013). The contribution of general cognitive abilities and number abilities to different aspects of mathematics in children. Journal of Experimental Child Psychology 116, 139156.

Träff, U., \& Passolunghi, M. C. (2015). Mathematical Skills in Children with Dyslexia. Learning and Individual Differences, 40, 108-114. Doi: 10.1016/j.lindif.2015.03.024

Vellutino, F. R., Fletcher, J. M., Snowling, M. J., \& Scanlon, D. M. (2004). Specific reading disability (dyslexia): What have we learned in the past four decades? Journal of Child Psychology \& Psychiatry, 45, 2-40. DOI: 10.1046/j.0021-9630.2003.00305.x

von Aster, M. G., \& Shalev, R. S. (2007). Number development and developmental dyscalculia. Developmental Medicine \& Child Neurology, 49, 868-873.

Vukovic, R.K., Lesaux, N.K., \& Siegel, L.S. (2010). The mathematics skills of children with reading difficulties. Learning and Individual Differences, 20, 639-643.

Wilson, A. J., \& Dehaene, S. (2007). Number sense and developmental dyscalculia. In D. Coch, G. Dawson, \& K. Fischer (Eds.), Human behavior, learning and the developing brain: Atypical development (pp. 212-238). New York, NY: Guilford Press. 
Wolf, M., \& Bowers, P. G. (1999). The Double-Deficit Hypothesis for the Developmental Dyslexias. Journal of Educational Psychology, 91, 415-438.

Wolf, M., Bowers, P. G., \& Biddle, K. (2000). Naming-speed processes, timing and reading: a conceptual review. Journal of Learning Disabilities, 33, 387-407. 
Table 1.

Background data of children in the dyslexia group and the control groups

\begin{tabular}{|c|c|c|c|c|c|c|}
\hline & \multicolumn{2}{|c|}{ Children with dyslexia } & \multicolumn{2}{|c|}{ Controls } & \multirow[b]{2}{*}{ Reliability } & \multirow[b]{2}{*}{ ANOVA/Chi-square-test } \\
\hline & $\mathrm{M}$ & SD & $\mathrm{M}$ & SD & & \\
\hline Age (in years) & 10.64 & 0.26 & 10.59 & 0.27 & & $F(1,53)=0.323, p=.572$ \\
\hline Word decoding* & 76.60 & 11.63 & 130.57 & 17.22 & $.97^{\mathrm{a}}$ & \\
\hline Text reading* & 5.95 & 2.19 & 12.60 & 2.22 & $.97^{\mathrm{a}}$ & $F(1,53)=115.02, p<.001$ \\
\hline Ravens Progressive & 24.80 & 3.72 & 26.31 & 3.68 & $.80^{\mathrm{b}}$ & $F(1,53)=2.13, p=.150$ \\
\hline \multicolumn{7}{|l|}{ Matrices* } \\
\hline $\mathrm{N}$ (number of girls) & $20(9)$ & & $35(17)$ & & & $\chi^{2}(1, N=55)=0.07, p=.80$ \\
\hline
\end{tabular}

Controls $=$ normal achievers

* Raw scores

${ }^{\text {a }}$ Split-half reliability, ${ }^{\mathrm{b}}$ Cronbach's alpha, 
Table 2.

Descriptive statistics for tasks by ability group

\begin{tabular}{|c|c|c|c|c|c|c|}
\hline \multirow[b]{2}{*}{ Tasks } & \multicolumn{2}{|c|}{ Children with dyslexia } & \multicolumn{2}{|c|}{ Controls } & \multirow[b]{2}{*}{ Reliability } & \multirow[b]{2}{*}{ ANOVA } \\
\hline & $\mathrm{M}$ & SD & $\mathrm{M}$ & SD & & \\
\hline Phonological segment subtraction & 6.60 & 3.33 & 11.54 & 2.23 & $.87^{\mathrm{a}}$ & $F(1,53)=43.42, p<.001, \omega^{2}=.44$ \\
\hline Color naming (sec) & 30.68 & 8.15 & 25.43 & 5.80 & $.90^{\mathrm{a}}$ & $F(1,53)=7.73, p<.001, \omega^{2}=.11$ \\
\hline Digit matching (sec) & 70.34 & 15.71 & 58.99 & 11.05 & $.79^{\mathrm{c}}$ & $F(1,53)=9.83, p=.003, \omega^{2}=.14$ \\
\hline Complex word span & 3.45 & 0.67 & 3.64 & 0.68 & $.85^{\mathrm{a}}$ & $F(1,53)=1.04, p=.314, \omega^{2}=.00$ \\
\hline Visual-matrix span & 3.90 & 0.97 & 3.50 & 1.44 & $.76^{\mathrm{a}}$ & $F(1,53)=1.22, p=.275, \omega^{2}=.00$ \\
\hline \multicolumn{7}{|l|}{ Symbolic number comparison } \\
\hline One digit (sec) & 0.84 & 0.18 & 0.72 & 0.11 & $.95^{\mathrm{a}}$ & $F(1,53)=8.86, p=.004, \omega^{2}=.13$ \\
\hline Two digit (sec) & 1.13 & 0.28 & 0.98 & 0.14 & $.79^{\mathrm{a}}$ & $F(1,53)=6.31, p=.015, \omega^{2}=.09$ \\
\hline \multicolumn{7}{|l|}{ Non-symbolic number comparison } \\
\hline Weber fraction & 0.23 & 0.06 & 0.25 & 0.14 & $.88^{\mathrm{a}}$ & $F(1,53)=0.46, p=.501, \omega^{2}=.01$ \\
\hline Response time (sec) & 0.86 & 0.21 & 0.78 & 0.17 & $.86^{\mathrm{a}}$ & $F(1,53)=2.13, p=.151, \omega^{2}=.02$ \\
\hline Arithmetic fluency & 32.10 & 10.45 & 42.06 & 12.85 & $.81^{\mathrm{a}}$ & $F(1,53)=8.70, p=.005, \omega^{2}=.12$ \\
\hline Multi-digit calculation & 6.00 & 2.18 & 8.23 & 1.91 & $.73^{\mathrm{a}}$ & $F(1,53)=15.64, p<.001, \omega^{2}=.21$ \\
\hline
\end{tabular}

${ }^{\mathrm{a}}$ Split-half reliability, ${ }^{\mathrm{b}}$ Cronbach's alpha, ${ }^{\mathrm{c}}$ Test-retest reliability 
Table 3.

Correlations among the tasks used in the study

\begin{tabular}{lcccccccccc} 
Tasks & 2 & 3 & 4 & 5 & 6 & 7 & 8 & 9 & 10 & 11 \\
\hline 1 Phonological segment subtraction & -.13 & -.46 & .03 & -.26 & -.11 & -.08 & -.16 & -.04 & .20 & .31 \\
2. Color naming & & .47 & -.27 & .08 & .36 & .36 & -.01 & .39 & -.43 & -.49 \\
3. Digit matching & & & -.33 & -.02 & .32 & .24 & .28 & .12 & -.34 & -.36 \\
4. Complex word span & & & & .26 & -.24 & -.27 & -.02 & -.09 & .37 & .15 \\
5. Visual-matrix span & & & & -.19 & -.02 & -.14 & .04 & .19 & .00 \\
6. One-digit NC & & & & & .77 & -.11 & .61 & -.55 & -.29 \\
7. Two-digit NC & & & & & & -.23 & .59 & -.52 & -.39 \\
8. Non-symbolic NC Weber fraction & & & & & & & & -.27 & .02 & -.16 \\
9. Non-symbolic NC RT & & & & & & & & -.30 & -.01 \\
10. Arithmetic fluency & & & & & & & & & .53 \\
11. Multi-digit calculation & & & & & & & & &
\end{tabular}

$\mathrm{n}=55$, correlation coefficients of $\mathrm{r}=.27$ or larger are significant at $p<.05$

$\mathrm{NC}=$ number comparison

$\mathrm{RT}=$ response time 